\section{Expresión disminuida de caspasa 3 asociada al polimorfismo del gen del antígeno-4 asociado a linfocito T-citotóxico (CTLA4) en pacientes chilenos con diabetes tipo 1}

\author{
BÁRBARA ANGEL B. ${ }^{1, a}$, ETHEL CODNER ${ }^{2}$, MIGUEL ARREDONDO O. ${ }^{3, \mathrm{~b}}$, \\ FRANCISCA SALAS P., ${ }^{4,}$, CAROLINA PIZARRO A. ${ }^{4, \mathrm{~d}}$, FRANCISCO PÉREZ B. ${ }^{4, \mathrm{~b}}$
}

\section{Decreased caspase 3 expression and cytotoxic T lymphocyte antigen-4 polymorphism in Chilean patients with type 1 diabetes}

Background: Several polymorphisms of the CTLA4 gene have been associated with autoimmune diseases. The activation of induced cell death is the major event and caspase 3 represents the main protein for the apoptotic machinery, especially in lymphocytes. Aim: To correlate CTLA4 polymorphisms with caspase 3 expression in peripheral blood mononuclear cells (PBMC) simulating in vitro the glucose effect. Material and Methods: CTLA4 polymorphisms were determined by restriction fragment length polymorphisms (RFLPs). PBMC from 21 patients with type 1 diabetes aged $8.5 \pm 4.3$ years and 21 healthy subjects aged $18.3 \pm 1.8$ years, were stimulated under normal ( $5 \mathrm{mM})$ and toxic $(14 \mathrm{mM})$ glucose conditions to assess its effect on the expression and activity of caspase 3. Relative abundance of caspase $3 \mathrm{mRNA}$ was measured by semi quantitative RT-PCR and its activity, by a colorimetric assay. Results: When stimulated with $14 \mathrm{mM}$ glucose, PBMC of G allele carriers with type 1 diabetes had significantly lower relative mRNA abundance of caspase 3 (median value $=0.12$, range 0.01-0.70 AU) compared with non-carriers (median value = 0.81, range 0.06-1.09 AU). When the incubation was carried out with the lower glucose concentration, a similar profile of caspase 3 activity was observed in diabetic patients carrying $G$ allele (median value $=0.57$, range 0.13-1.20 AU) as compared with non-carriers (median value $=0.89$, range 0.14-5.50 AU). No significant changes after stimulating with glucose, were observed in PBMCs of the control group. Conclusions: PBMC of recently diagnosed patients with T1D, carrying the G allele in $+49 A / G$ polymorphisms of CTLA4, have a decreased expression and activity of caspase 3.

(Rev Med Chile 2012; 140: 1109-1115).

Key words: Apoptosis; Caspase 3; Diabetes Mellitus, type 1.

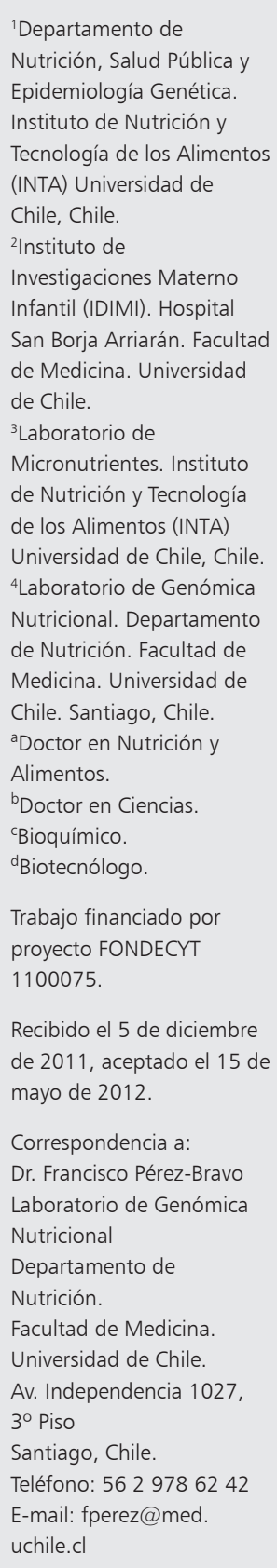

$\mathrm{L}$ a incidencia de la diabetes tipo 1 (DM1) en Santiago, Chile se ha incrementado en los $\checkmark$ últimos años, lo que es concordante con lo observado en América Latina como a nivel mundial ${ }^{1,2}$.

La DM1 es una enfermedad autoinmune donde células $\mathrm{T}$ destruyen selectivamente a las células $\beta$ pancreáticas productoras de insulina ${ }^{3-5}$. Las células T activadas invaden los islotes, produciendo un fenómeno denominado "insulitis". La destrucción de los islotes está mediada por una compleja interacción entre los linfocitos activados, 
citoquinas y macrófagos ${ }^{5-7}$. La apoptosis es un proceso fundamental que está involucrado en la viabilidad y en la destrucción tanto de las células T como de las células $\beta$. Se ha observado que la apoptosis es un fenómeno crucial para activar a las células $T$ específicas, que agreden al páncreas y que dan cuenta de la aparición de la enfermedad ${ }^{5}$. La disfunción de la apoptosis se ha relacionado con la aparición de diversas enfermedades autoinmunes. Diversos estudios han propuesto que el bloqueo de la apoptosis estaría involucrado en la patogénesis de estas enfermedades ${ }^{5,8}$.

El gen CTLA4 contiene 4 exones y se han descrito varios polimorfismos en la región promotora $^{9-12}$. La sustitución de una $G$ por una $A$ en la posición 49 del exón $1(+49 \mathrm{~A} / \mathrm{G})$, causa un cambio de una treonina por una alanina en el codón 17 del péptido señal (A17T), la presencia de este alelo ha sido asociada a la predisposición a desarrollar enfermedades autoinmunes ${ }^{13-15}$. Diversos autores han explicado que esta asociación se relaciona con bajos niveles de ARNm de la forma soluble de la proteína CTLA4, teniendo un papel funcional en la susceptibilidad para desarrollar enfermedades autoinmunes.

Las caspasas son cisteína-aspartil proteasas específicas conservadas evolutivamente que juegan un papel clave en la apoptosis ${ }^{16}$. La apoptosis cursa a través de dos vías principales: la vía extrínseca o la vía del receptor de muerte celular que involucra la superfamilia de receptores del factor de necrosis tumoral (dependiente de la proteína CD95 o Fas) y la vía intrínseca o mitocondrial. La caspasa 8 y 9 son proteínas que están involucradas tanto en la vía extrínseca como intrínseca, mientras que las caspasas 3, 6, y 7 son proteínas efectoras ${ }^{17}$. Se ha reportado la existencia de alteraciones en la apoptosis en células T periféricas en sujetos afectados por enfermedades autoinmunes ${ }^{18}$.

En ratones deficientes en la proteína Fas, se ha observado una activación de la muerte celular inducida, evento que contribuye al desarrollo de autoinmunidad ${ }^{19}$. En el ratón diabético no-obeso (NOD), modelo de diabetes autoinmune, se ha demostrado una resistencia a las señales apoptóticas en los linfocitos T periféricos ${ }^{20-22}$. Además, estudios recientes describen una expresión alterada de Fas y su ligando (FasL), y una expresión reducida de las caspasas 8 y 3 en los linfocitos T periféricos, tanto en los modelos animales como en pacientes con $\mathrm{DM} 1^{6,7}$. Considerando que hasta la fecha no hay estudios que relacionen la expresión diferencial de caspasa 3 con genotipos específicos de CTLA4 en la DM1, el propósito de este estudio fue caracterizar la expresión y la actividad de caspasa 3 en un modelo de CMPs provenientes de pacientes con DM1 y controles sometiendo a está células a dos estímulos de glucosa (normal y tóxica) y evaluando su efecto sobre la expresión y la actividad de caspasa 3.

\section{Materiales y Métodos}

\section{Sujetos}

El estudio celular se realizó con 21 pacientes con DM1 (14 mujeres, 7 hombres; edad promedio $8,5 \pm 4,3$ años) menores de 15 años con diagnóstico reciente de DM1 debutantes en el IDIMI y provenientes de comunas de nivel socioeconómico medio-bajo. La muestra fue tomada en un período no mayor a los quince días a partir del diagnóstico (según criterio Asociación Americana de Diabetes, ADA). El grupo control correspondió a 21 individuos sanos (12 mujeres, 8 hombres; edad promedio $18,3 \pm 1,8$ años), sin antecedentes familiares de diabetes, ni enfermedades autoinmunes provenientes de dos comunas de nivel socioeconómico medio-bajo. Se escogieron jóvenes mayores a los pacientes con DM1 para descartar la posibilidad de DM1 en este grupo. Este protocolo fue aprobado por los comités de ética de la Facultad de Medicina de la Universidad de Chile e IDIMI respectivamente. Todos los participantes y/o sus padres firmaron el correspondiente consentimiento informado.

\section{Estudio experimental}

El ADN genómico fue extraído de sangre periférica utilizando un protocolo estándar (Winkler, Santiago, Chile). Los genotipos para el gen CTLA4 fueron obtenidos mediante la amplificación del ADN con PCR convencional y análisis de fragmentos de restricción. Se amplificó una región de 162 pb localizado en el exón 1 del gen CTLA4 con los partidores: sentido 5'-GCTCTACTTCCTGAAGACCT-3'; antisentido 5'-AGTCTCACTCACCTTTGCAG-3. La reacción de PCR fue realizada en $20 \mu \mathrm{L}$ de reacción con 5 pmoles de cada partidor, 50 ng de DNA genómico, $200 \mu \mathrm{M}$ de cada dNTP y $0,5 \mathrm{U}$ de Taq-Polimerasa. La reacción se estandarizó en un período de $7 \mathrm{~min}$ a $95^{\circ} \mathrm{C}$ para la activación de la enzima y 40 ciclos 
que consistieron en denaturación del $\mathrm{ADN}$ a $95^{\circ} \mathrm{C}$ por $30 \mathrm{~s}$, unión de los partidores a $56^{\circ} \mathrm{C}$ por $30 \mathrm{~s}$ y extensión a $72^{\circ} \mathrm{C}$ por $30 \mathrm{~s}$, cn un período de extensión final a $72^{\circ} \mathrm{C}$ durante $15 \mathrm{~min}$. El análisis de fragmentos de restricción se realizó en $5 \mu \mathrm{L}$ de producto incubado a $37^{\circ} \mathrm{C}$ durante toda la noche con $0,5 \mathrm{U}$ de la enzima BvbI. El producto de digestión (162 pb, 90 bp y 74 bp) fue visualizado en geles de agarosa al 2,5\% con bromuro de etidio ${ }^{23}$.

\section{Separación y cultivo celular}

Las CMPs fueron aisladas a partir de sangre heparinizada mediante centrifugación en gradiente de densidad Ficoll-Hystopaque (densidad: 1,119; Sigma Diagnostic, St. Louis, MO), lavadas dos veces con el medio RPMI 1640 sin glucosa (GibcoBRL, Grand Island, NY, USA). Las células fueron incubadas con glucosa ( 5 y $14 \mathrm{mM}$ ) o sin glucosa por $20 \mathrm{~h}$, a $37^{\circ}, 5 \% \mathrm{CO}_{2}$ y $80 \%$ de humedad, en placas Petri de 6 pocillos con medio RPMI 1.640 sin glucosa, suplementado con suero fetal bovino al 10\% fetal y antibióticos en duplicado (a una concentración de 1 x $10^{6}$ células en $1 \mathrm{ml}$ de medio de cultivo). La recuperación de las células fue llevada a cabo mediante una centrifugación a baja velocidad (3.000 rpm x $5 \mathrm{~min}$ ) y un tratamiento suave con tripsina-EDTA a $37^{\circ} \mathrm{C}$ por $5 \mathrm{~min}$. Las células fueron lavadas con PBS y la viabilidad celular fue determinada utilizando una tinción de azul Tripán en una cámara de Neubauer. Una alícuota fue removida para la extracción de proteínas y otra para la extracción de ARNm. Estas alícuotas fueron almacenadas y congeladas a $-80^{\circ}$ ${ }^{\circ} \mathrm{C}$ en DMSO al $10 \%$.

\section{Expresión de ARNm para Fas y caspasa 3}

La abundancia relativa del ARNm de Fas y caspasa 3 fue semicuantificada mediante RT-PCR utilizando partidores múltiples. El ARNm total fue extraído con Trizol (Gibco-BRL) y la síntesis de ADNc se llevó a cabo utilizando $2 \mu$ g de cada muestra de ARNm con hexámeros al azar (Random Partidores) con $200 \mathrm{U}$ de enzima Superscript II (Gibco-BRL); posteriormente 200 ng de la alícuota de ADNc fue amplificada en un volumen de reacción de $100 \mu \mathrm{L}$ con 20 pmol de los partidores, 2,5 U Taq ADN polimerasa (Gibco-BRL), 200 $\mathrm{mmol} / \mathrm{l}$ de cada deoxinucleósido trifosfato y 1,5 $\mathrm{mmol} / \mathrm{l} \mathrm{MgCl}_{2}$. Las secuencias de los partidores para caspasa 3 fueron: sentido 5'-ATG GAG AAC ACT GAA AAC TCA GTG-3', anti-sentido 5'-
TAG TGA TAA AAA TAG AGT TCT TTT G-3’ y para Fas fueron: 5' GCCCAAGTGACTGACATCA 3'y anti-sentido: 5' ACTGTGCAGTCCCTAGCTT 3’. La expresión de $\beta$-actina se utilizó como un control de ARNm empleando los siguientes partidores que generaron un producto de 354 pb: sentido 5'-ACC AAC TGG GAC GAC ATG GAG-3', anti-sentido 5'-CGT GAG GAT CTT CAT GAG GTA GTC-3. Se utilizaron 30 ciclos para caspasa-3-Fas y 25 ciclos para $\beta$-actina. Todos los productos de PCR fueron analizados por electroforesis en gel de agarosa al 1,2\% mediante un escaneo densitométrico del gel utilizando el software Bio-Capt versión 11.01 y los resultados han sido expresados en una razón caspasa-3/ $\beta$-actina o Fas/ $\beta$-actina. Para la determinación de proteínas, las muestras fueron procesadas usando un buffer de lisis celular contenido en el kit (ApoTargetTM Casp 3, Biosurce International, Inc. California, USA) y cuantificadas por método de Lowry.

La actividad de caspasa 3 (200 mg de proteína) medida con un kit colorimétrico (ApoTargetTM Casp-3, Biosurce International, Inc, California, USA) con DEVD-pNA (p-Nitroaniline) como sustrato. Este enfoque nos permite determinar la actividad in vitro a través de la medición proteolítica de caspasa3 inducida en nuestro experimento por glucosa, midiendo la absorbancia a $405 \mathrm{~nm}$. El incremento en la actividad de caspasa 3 fue determinado por comparación directa con las lecturas en cada condición ( $5 \mathrm{mM}$ y $14 \mathrm{mM}$ de estímulo de glucosa) y con las lecturas de los controles (células sin estímulos de glucosa).

\section{Análisis estadístico}

Se utilizó test de Kruskal-Wallis para estimar la expresión relativa de Fas entre DM1 y controles. El test de Friedman y Wilcoxon para comparar la expresión y actividad de caspasa 3 y de acuerdo con los genotipos $+49 \mathrm{~A} / \mathrm{G}$ del gen CTLA4 en los diferentes estímulos de glucosa.

\section{Resultados}

\section{Expresión de Fas}

Para evaluar la presencia de un patrón diferencial de apoptosis en CMPs se realizó una determinación de la expresión de Fas bajo la influencia de la hemaglutinina que induce apoptosis vía Fas-Fas-ligando. El efecto de apoptosis inducida 
Expresión disminuida de caspasa 3 y polimorfismo CTLA4 en DM1 - B. Angel et al

en las CMPs con hemaglutinina, mostró que los DM1 presentaron una expresión diferencial de Fas (Figura 1), en ambas condiciones de glucosa (5 mM y $14 \mathrm{mM}$ ).

\section{Expresión de caspasa 3 en función del genotipo CTLA4}

Se analizó la expresión del ARNm de caspasa 3 y los datos se dividieron en genotipo AA y en portadores del alelo $G(A G+G G)$ según el polimorfismo +49 A/G del gen CTLA4. Los resultados obtenidos en pacientes y controles, mostraron que la expresión relativa del ARNm de caspasa 3 aumentó en relación a $\beta$-actina cuando la concentración de glucosa se incrementó $(\mathrm{p}=0,06)$ para el genotipo AA. En cambio, los pacientes con DM1 portadores del alelo $\mathrm{G}$ (AG+GG) mostraron una disminución significativa en la relación ARNm caspasa 3/ARNm $\beta$-actina $(\mathrm{p}=0,02)$ (Figura 2). Los niveles de expresión del ARNm de caspasa 3 se mantuvieron sin cambios en los controles.

Al comparar la abundancia relativa del ARNm de caspasa 3 en cada punto de exposición a glucosa, entre portadores y no portadores del alelo $G$ en el grupo DM1 se observó que en la condición de glucosa $14 \mathrm{mM}$, los portadores del alelo $\mathrm{G}$ mostraron una menor abundancia relativa (mediana de los valores de expresión $=0,12$ dentro del rango [0,01-0,70]), en comparación con los no portadores (mediana de los valores de expresión $=$
$0,81$ dentro del rango $[0,06-1,09]) ; \operatorname{con} p=0,031$. El mismo análisis para el grupo control no mostró diferencias en la abundancia relativa del ARNm de caspasa 3 entre los genotipos o en las distintas condiciones de glucosa.

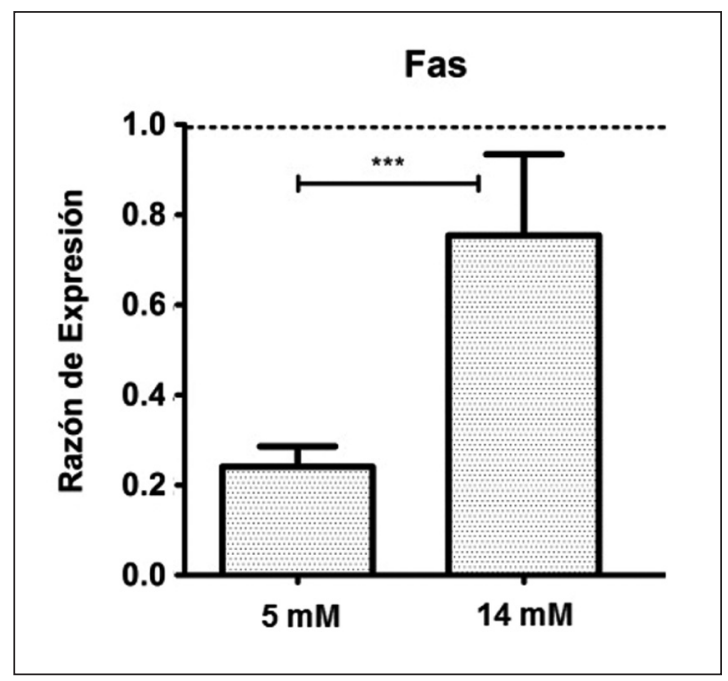

Figura 1. Efecto de glucosa sobre la expresión génica de Fas. Razones de expresión del gen Fas en el grupo de pacientes con DM1 $(n=21)$ en relación a los controles $(n=21)$. Valores mayores a 1 indican aumento de la expresión. Significancia estadística según test de Kruskal-Wallis. ${ }^{* *} p<0,001$.

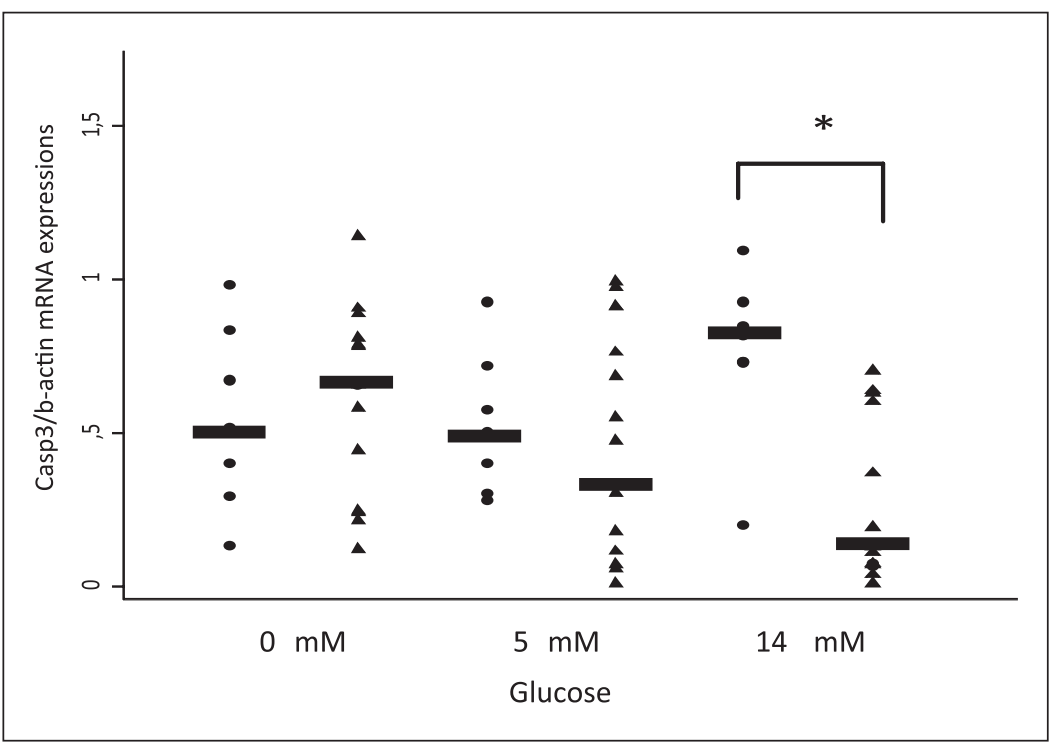

Figura 2. Expresión del ARNm de caspasa $3 / \beta$-actina en CPMs. Se muestran los no portadores (círculos) y portadores (triángulos) del alelo $\mathrm{G}$ según el estímulo de glucosa. Se observa una disminución significativa en los portadores de este alelo en la condición de 14 $m M$ de glucosa $(p=0,03)$. 


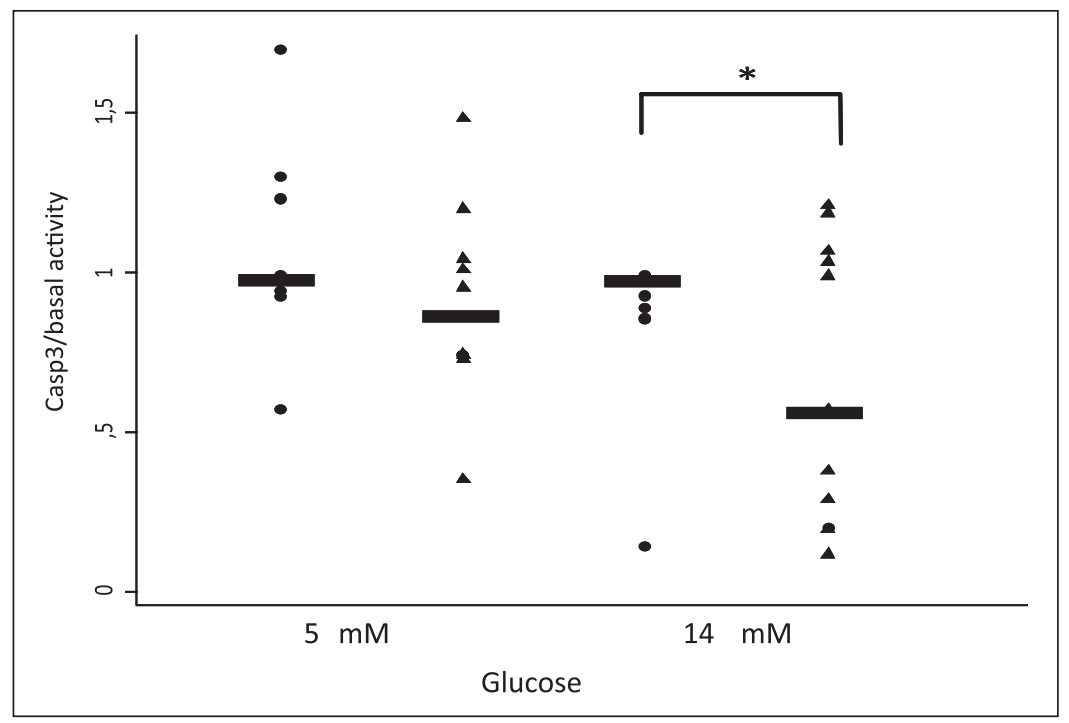

Figura 3. Actividad caspasa 3/basal en las CMPs, los no portadores (círculos) y portadores (triángulos) del alelo $G$ de acuerdo con el estímulo de glucosa. Se observó una disminución significativa en los portadores de este alelo en la condición de $14 \mathrm{mM}$ de glucosa $(p=0,03)$.

\section{Actividad de caspasa 3 en función del genotipo CTLA4}

Este análisis se realizó con 200 mg de proteína de CMPs en respuesta a diferentes concentraciones de glucosa. Los resultados fueron divididos por genotipo como AA y portadores del alelo $G$ (AG+GG) según polimorfismo CTLA4 (Figura 3). Nuestros datos mostraron que esta actividad no tuvo variación con el incremento en la concentración de glucosa $(\mathrm{p}=0,25)$. En los portadores del alelo $\mathrm{G}(\mathrm{AG}+\mathrm{GG})$ con DM1, la actividad caspasa 3 mostró una tendencia decreciente con el aumento de la concentración de glucosa. Se observó de forma similar que en el perfil de expresión de caspasa 3 en la condición de $14 \mathrm{mM}$ de glucosa, que los pacientes portadores del alelo $\mathrm{G}$ mostraron una menor actividad (mediana de los valores de expresión (unidad arbitraria) $=0,57$ dentro del rango $[0,13-1,2])$ en comparación con los DM1 no portadores (mediana de los valores de expresión $=0,887$ dentro del rango $[0,14-5,5])$, con una diferencia estadísticamente significativa $(\mathrm{p}=0,03)$. En el grupo control, la actividad de la caspasa 3 no varió entre portadores y no portadores en relación a las concentraciones de glucosa.

\section{Discusión}

La confirmación de los roles de los genes CTLA4 y el receptor de la proteína fosfatasa lin- foide (PTPN2) como reguladores de la inmunomodulación negativa de las células $T$ a través de las células $\mathrm{T}$ regulatorias, revela la complejidad de la respuesta inmune observada en la DM124.

Las moléculas CTLA4 y PTPN22, controlan la activación de células $\mathrm{T}$ y a diferencia de CD28, CTLA4 entrega señales negativas a las células $\mathrm{T}^{25}$. Varios polimorfismos del gen CTLA4 han sido relacionados a una función inhibitoria reducida sugiriendo que estos podrían jugar un papel importante en varias etapas regulatorias de las células $\mathrm{T}^{9,26-28}$. Estudios previos de nuestro grupo en polimorfismos de CTLA4 mostraron una potencial reducción de la proteína circulante CTLA4 en pacientes con DM1 portadores del alel G ${ }^{29}$, fenómeno que Purohit y cols ${ }^{30}$, no habían detectado en sus estudios previos.

Los datos presentados en este trabajo muestran una posible relación entre el polimorfismo +49 A/G del gen CTLA4 y un marcador de apoptosis como caspasa 3. La apoptosis parece jugar un rol central en los mecanismos moleculares que conducen a la autoinmunidad ${ }^{31,32}$. Aunque las células $\mathrm{T}$ colaboradoras representan el principal componente en la insulitis autoinmune, la respuesta humoral y de células policlonales encontradas en pacientes con DM1 sugieren la participación de otros múltiples eventos dirigidos hacia células $\beta$ pancreáticas, entre los cuales la apoptoisis parece jugar un papel fundamental ${ }^{33,34}$. 
Expresión disminuida de caspasa 3 y polimorfismo CTLA4 en DM1 - B. Angel et al

Vendrame y cols ${ }^{35}$ reportaron una expresión alterada de caspasa 3 en pacientes con DM1. Sus resultados mostraron una resistencia a la apoptosis mediada por el receptor de membrana Fas debido, al menos en un grupo de pacientes, a un defecto en la expresión de caspasa 3 (principal ejecutor de la muerte celular por apoptosis dependiente de Fas). Ellos propusieron que la expresión y función defectuosa de caspasa 3 en linfocitos periféricos de pacientes con DM1 u otras enfermedades autoinmunes como tiroiditis, puede cumplir una función importante en la resistencia a la muerte celular y así contribuir al desarrollo de autoinmunidad en individuos susceptibles genéticamente ${ }^{35,36}$.

Nuestros resultados indican que las CPMs provenientes de pacientes portadores del alelo $\mathrm{G}$ del polimorfismo $+49 \mathrm{~A} / \mathrm{G}$ de CTLA4 mostraron una expresión reducida de caspasa 3 después de la exposición a altos niveles de glucosa. Este resultado es concordante con lo observado por Vendrame y cols ${ }^{35}$ y podría indicar una resistencia a la muerte celular por parte del linfocito T.

Un aspecto importante a considerar en la interpretación de nuestros resultados tiene relación con la posible influencia de la edad sobre los marcadores de apoptosis, en particular sobre caspasa 3. En nuestro estudio, los pacientes con DM1 son más jóvenes que los controles, esto debido a que la búsqueda de controles mayores es necesaria para descartar la presencia de DM1. Sin embargo, esta diferencia de edad podría estar afectando la actividad de caspasa 3. En otros modelos celulares (cardiomiocitos en cultivo, células progenitoras endoteliales y músculo) se ha observado que la concentración de caspasa 3 está aumentada y que su actividad tiende a ser mayor con la edad ${ }^{37-39}$. Este antecedente es relevante y a pesar de que no hay antecedentes en CMPs y que los controles también son jóvenes, debiera interpretarse con cautela.

Nuestros datos apoyarían el papel esencial del gen CTLA4 en la inmuno-modulación de las células T. En particular, este es el primer reporte sobre la relación entre el polimorfismo $+49 \mathrm{~A} / \mathrm{G}$ del gen CTLA4 y la activación de caspasa 3. Recientemente, se ha mostrado la importancia de la activación de las caspasas en patologías mediadas por inmunidad $^{20}$. Además, nuestros resultados muestran que la presencia del alelo $\mathrm{G}$ del polimorfismo +49 A/G de CTLA4 en CMPs de niños con DM1 podría estar relacionada a una relativa baja en la expresión y actividad de caspasa 3 en condiciones de estrés por glucosa. Esto sugiere una resistencia a la apoptosis por parte de las CMPs, produciéndose dos efectos conjuntos: una mayor sobrevida celular y por lo tanto, mayor probabilidad de infiltración y un efecto de masa celular lo que condicionaría una mayor producción de citoquinas. En ambos casos el escenario se corresponde con una mayor agresividad de las células $\mathrm{T}$ circulantes.

\section{Referencias}

1. Carrasco E, Pérez-Bravo F, Santos JL, López G, Calvillán $\mathrm{M}$, Wolff $\mathrm{C}$, et al. One of the lowest validated incidence rates of insulin dependent diabetes mellitus in the Americas: Santiago, Chile. Diabetes Res Clin Pract 1996; 34: S153-7.

2. Carrasco E, Pérez-Bravo F, Dorman J, Mondragón A, Santos JL. Increasing incidence of type 1 diabetes in population from Santiago of Chile: trends in a period of 18 years (1986-2003). Diabetes Metab Res Rev 2006; 22: 34-7.

3. Alizadeh BZ, Koeleman BP. Genetic polymorphisms in susceptibility to Type 1 Diabetes. Clin Chim Acta 2008; 387: 9-17.

4. Anderson MS, Bluestone JA. The NOD mouse: a model of immune dysregulation. Annu Rev Immunol 2005; 23 : 447-85.

5. Pirot P, Cardozo AK, Eizirik DL. Mediators and mechanisms of pancreatic beta-cell death in type 1 diabetes. Arq Bras Endocrinol Metabol 2008; 52: 156-65.

6. Gregersen PK, Behrens TW. Genetics of autoimmune diseases-disorders of immune homeostasis. Nat Rev Genet 2006; 7: 917-28.

7. Atkinson MA, Bluestone JA, Eisenbarth GS, Hebrok M, Herold KC, Accili D, et al. How does type 1 diabetes develop?: the notion of homicide or beta-cell suicide revisited. Diabetes 2011; 60: 1370-9.

8. Pearl-Yafe M, Yolcu ES, Yaniv I, Stein J, Shirwan H, Askenasy N. The dual role of Fas-ligand as an injury effector and defense strategy in diabetes and islet transplantation. Bioessays 2006; 28: 211-22.

9. Kouki T, Gardine CA, Yanagawa T, Degroot LJ. Relation of three polymorphisms of the CTLA- 4 gene in patients with Graves' disease. J Endocrinol Invest 2002; 25: 208-13.

10. Lee YJ, Lo FS, Shu SG, Wang CH, Huang CY, Liu HF, et al. The promoter region of the CTLA4 gene is associated with type 1 diabetes mellitus. J Pediatr Endocrinol Metab 2001; 14: 383-8.

11. Van Belle TL, Coppieters KT, von Herrath MG. Type 1 diabetes: etiology, immunology, and therapeutic strate- 
gies. Physiol Rev. 2011; 91: 79-118.

12. Turpeinen H, Laine AP, Hermann R, Simell O, Veijola R, Knip M, et al. A linkage analysis of the CTLA4 gene region in Finnish patients with type 1 diabetes. Eur J Immunogenet 2003; 30: 289-93.

13. Teft WA, Kirchhof MG, Madrenas J. A molecular perspective of CTLA-4 function. Annu Rev Immunol 2006; 24: 65-97.

14. Ueda H, Howson JM, Esposito L, Heward J, Snook H, Chamberlain G, et al. Association of the T-cell regulatory gene CTLA4 with susceptibility to autoimmune disease. Nature 2003; 423: 506-11.

15. Vaidya B, Pearce S. The emerging role of the CTLA-4 gene in autoimmune endocrinopathies. Eur J Endocrinol 2004; 150: 619-26.

16. Reed JC. Mechanisms of apoptosis. Am J Pathol 2000; 157: 1415-30

17. Siegel RM. Caspases at the crossroads of immune-cell life and death. Nat Rev Immunol 2006; 6: 308-17.

18. Opferman JT. Apoptosis in the development of the immune system. Cell Death Differ 2008; 15: 234-42.

19. Nagata S, Suda T. Fas and Fas ligand: lpr and gld mutations. Immunol Today 1995; 16: 39-43.

20. Augstein P, Bahr J, Wachlin G, Heinke P, Berg S, Salzsieder E, et al. Cytokines activate caspase-3 in insulinoma cells of diabetes-prone NOD mice directly and via upregulation of Fas. J Autoimmun 2004; 23: 301-9.

21. Reddy S, Bradley J, Ginn S, Pathipati P, Ross JM. Immunohistochemical study of caspase-3-expressing cells within the pancreas of non-obese diabetic mice during cyclophosphamide-accelerated diabetes. Histochem Cell Biol 2003; 119: 451-61.

22. Yamada K, Ichikawa F, Ishiyama-Shigemoto S, Yuan $\mathrm{X}$, Nonaka K. Essential role of caspase-3 in apoptosis of mouse beta-cells transfected with human Fas. Diabetes 1999; 48: 478-83.

23. Balic I, Angel B, Codner E, Carrasco E, Pérez-Bravo F. Association of CTLA-4 polymorphisms and clinicalimmunologic characteristics at onset of type 1 diabetes mellitus in children. Hum Immunol 2009; 70: 116-20.

24. Duffy DL. Genetic determinants of diabetes are similarly associated with other immune-mediated diseases. Curr Opin Allergy Clin Immunol 2007; 7: 468-74.

25. Liadis N, Murakami K, Eweida M, Elford AR, Sheu L, Gaisano H, et al. Caspase-3-dependent beta-cell apoptosis in the initiation of autoimmune diabetes mellitus. Mol Cell Biol 2005; 25: 3620-9.

26. Ligers A, Teleshova N, Masterman T, Huang WX, Hillert J. CTLA-4 gene expression is influenced by promoter and exon 1 polymorphisms. Genes Immun 2001; 2: 145-52.

27. Maurer M, Loserth S, Kolb-Maurer A, Ponath A, Wiese S,
Kruse N, et al. A polymorphism in the human cytotoxic T-lymphocyte antigen 4 (CTLA4) gene (exon $1+49)$ alters T-cell activation. Immunogenetics 2002; 54: 1-8.

28. van der Merwe PA, Davis SJ. Molecular interactions mediating $\mathrm{T}$ cell antigen recognition. Annu Rev Immunol 2003; 21: 659-84.

29. Momin S, Flores S, Angel BB, Codner DE, Carrasco PE, Pérez-Bravo F. Interactions between programmed death 1 (PD-1) and cytotoxic T lymphocyte antigen 4 (CTLA4) gene polymorphisms in type 1 diabetes. Diabetes Res Clin Pract 2009; 83: 289-94.

30. Purohit S, Podolsky R, Collins C, Zheng W, Schatz D, Muir A, et al. Lack of correlation between the levels of soluble cytotoxic T-lymphocyte associated antigen-4 (CTLA-4) and the CT-60 genotypes. J Autoimmune Dis 2005; $2: 8$.

31. Ou D, Wang X, Metzger DL, James RF, Pozzilli P, Plesner A, et al. Synergistic inhibition of tumor necrosis factor-related apoptosis-inducing ligand-induced apoptosis in human pancreatic beta cells by Bcl-2 and X-linked inhibitor of apoptosis. Hum Immunol 2005; 66: 274-84.

32. Pearce SH, Merriman TR. Genetic progress towards the molecular basis of autoimmunity. Trends Mol Med 2006; 12: 90-8.

33. Hildeman DA, Zhu Y, Mitchell TC, Kappler J, Marrack P. Molecular mechanisms of activated $\mathrm{T}$ cell death in vivo. Curr Opin Immunol 2002; 14: 354-9.

34. Purohit S, She JX. Biomarkers for type 1 diabetes. Int J Clin Exp Med 2008; 1: 98-116.

35. Vendrame F, Santangelo C, Misasi R, Dionisi S, Gizzi C, Realacci M, et al. Defective lymphocyte caspase-3 expression in type 1 diabetes mellitus. Eur J Endocrinol 2005; 152: 119-25.

36. Vendrame F, Segni M, Grassetti D, Tellone V, Augello G, Trischitta V, et al. Impaired caspase- 3 expression by peripheral $\mathrm{T}$ cells in chronic autoimmune thyroiditis and in autoimmune polyendocrine syndrome-2. J Clin Endocrinol Metab 2006; 91: 5064-8.

37. Kakarla SK, Rice KM, Katta A, Paturi S, Wu M, Kolli $\mathrm{M}$, et al. Possible molecular mechanism underlying age-related cardiomyocyte apoptosis in the F344BN rat heart. J Gerontol A Biol Sci Med Sci 2010; 65: 147-55.

38. Leeuwenburgh C, Gurley CM, Strotman BA, DupontVersteegden EE. Age-related differences in apoptosis with disuse atrophy in soleus muscle. Am J Physiol Regul Integr Comp Physiol 2005; 288: 1288-96.

39. Kushner EJ, MacEneaney OJ, Weil BR, Greiner JJ, Stauffer BL, DeSouza CA. Aging is associated with a proapoptotic endothelial progenitor cell phenotype. J Vasc Res 2011; 48: 408-14. 